\title{
Deepfake Warnings for Political Videos Increase Disbelief but Do Not Improve Discernment: Evidence from Two Experiments*
}

\author{
John Ternovskił, Joshua Kalla, ${ }^{*}$ Peter M. Aronow ${ }^{\diamond}$ \\ January 14, 2021 \\ PRELIMINARY
}

\begin{abstract}
Recent advances in machine learning have led to the development of the "deepfake," a convincingly realistic, computer-generated video of a public figure saying something they have not actually said. Policymakers have expressed concern that deepfakes could mislead voters and affect election outcomes, but existing research has found minimal persuasive effects. In this paper, we explore a downstream consequence of deepfakes: if voters are repeatedly warned of the existence and dangers of deepfakes, they may simply begin to distrust all political video footage - whether real or fake. Through two online survey experiments, we found that voters were unable to discriminate between a real video and a deepfake. Statements warning about the existence of deepfakes did not enhance participants' ability to successfully spot manipulated video content. Instead, these warnings consistently induced participants to believe that the videos they watched were fake, even when the videos were real. The warnings were not specific to the video participants were watching; simply stating that deepfakes exist increased distrust of any accompanying video. Our findings suggest that even if deepfakes are not themselves persuasive, rhetoric about deepfakes can nevertheless be weaponized by politicians and campaigns to dismiss and disown real videos.
\end{abstract}

\footnotetext{
* This research was approved by the Yale University IRB. We thank Gregory A Huber, David G. Rand, and Todd Rogers for useful discussions. We are grateful to Grace Kang and Rosa Kleinman for their research assistance. The statements made and views expressed are solely the responsibility of the authors. JT was responsible for all computing, data collection and analysis, and wrote the initial manuscript and supplementary materials. All authors contributed to conceptualization, study design, and revision of the manuscript.

† Ph.D. candidate, Department of Political Science, Yale University. john.ternovski@yale.edu.

¥ Assistant Professor, Departments of Political Science and Statistics \& Data Science, Yale University. josh.kalla@yale.edu.

$\checkmark$ Associate Professor, Departments of Political Science, Biostatistics, and Statistics \& Data Science, Yale University. peter.aronow@yale.edu.
} 
Technological advances have begun to change critical aspects of democratic politics. The increased connectedness of our systems of communication has led to a world where political misinformation disseminates more rapidly than ever (Vosoughi, Roy, \& Aral, 2017). Breakthroughs in machine learning have led to the development of software that can seamlessly fabricate videos of any given individual. A video can be made in which a politician appears to say something they have never actually said in real life. These computer-manipulated videos are commonly known as "deepfakes."

Policymakers have raised concerns that deepfakes may mislead voters and sway election outcomes. For example, during a hearing of the United States House of Representatives Intelligence Committee, Adam Schiff, the Committee's chair, noted that deepfakes allow "malicious actors to foment chaos, division or crisis," and that such videos "have the capacity to disrupt entire campaigns, including that for the presidency" (O’Sullivan, 2019, p. 1). Computer and social scientists have echoed these concerns (e.g., Dack, 2019). However, recent randomized experiments on the impacts of deepfakes in American politics have found no evidence that people believe the content of the manipulated videos (Wittenberg, Zong, \& Rand, 2020; Vaccari \& Chadwick, 2020). More recently, Barari, Lucas \& Munger (2021) did find that deepfakes were persuasive but their effects were comparable to that of textual misinformation. ${ }^{1}$

In this paper, we explore a downstream consequence of deepfakes: do warnings of the existence and dangers of deepfakes cause voters to distrust all political video footage, whether real or fake? For example, in January 2021, supporters of Donald Trump suggested that a video Trump shared via Twitter in which he conceded the 2020 election was a deepfake (Villareal, 2021). Might politicians use the existence of deepfakes to call into doubt real videos that paint them in a negative light? A robust literature on textual misinformation (for a review, see Lazer et al., 2018) has found that elite discourse about "fake news" may lower trust in the media and prime participants to disbelieve the veracity of real news (Van Duyn \& Collier, 2019). Official warnings about fake news similarly induce participants to disbelieve true headlines (Clayton et al. 2019; Pennycook, Bear et al., 2020).

Across two studies, we demonstrate similar findings about deepfake warnings. The first study used an actor posing as a politician sharing an extreme policy position. Using a factorial design, participants were randomized in a first factor to either see a real video of the politician or a deepfake version of the video and, in a second factor, to receive a warning about deepfakes or no warning. The second study made use of Americans' low levels of policy knowledge (e.g., Barabas et al., 2014, Lenz, 2012) to show a real video of a real-world politician making a policy statement that is atypical of his party and is not widely known (and thus might be believed to be a deepfake). Both studies measured belief in the content of the videos and trust in video as a source of political information.

We found that participants were unable to discriminate between real and deepfaked video even when they were warned about the existence of deepfakes. Instead, deepfake warnings induced participants to disbelieve any associated political video - real or fabricated. In other words, a general statement warning about deepfakes was enough to nudge participants to disbelieve real video clips of politicians making policy statements. The effects were large and consistent across both studies. That said, we found no evidence that such warnings made participants distrust video as a source of

\footnotetext{
1 There is also a recent deepfake study in the Netherlands that found modest persuasive effects among a subset of participants (Dobber at al., 2020).
} 
political information more broadly; we saw precisely-estimated null effects on more general survey measures of trust in video footage of politicians.

This paper is organized as follows. First, we present a brief overview of the data and the designs of both survey experiments. Next, we report our main findings and discuss the implications of the results. We conclude with broader policy implications and delineate avenues for further research.

\section{Experimental Design}

We ran two pre-registered online survey experiments on Lucid Theorem ${ }^{2}$ in the spring and fall of 2020. The full survey questionnaires are provided in the Supplementary Materials (Appendix).

\section{Study 1: Fictional Politician ${ }^{3}$}

We created a video of an actor playing a fictional politician advocating an extreme policy position: support for a law requiring doctors to use essential oils to treat cancer before attempting treatments with conventional medicine. (A screenshot of the video can be viewed in Figure 1, left.) The actor also recorded another video that we used as training footage for our deepfake, where he recited a generic political speech. ${ }^{4}$ We then used Amazon's graphics-accelerated Elastic Computing (EC2) environment to parse the training footage into a training set of still .jpg images of the actor. These images were used to build a deepfake model using a widely available, open-source deepfake software package, DeepFaceLab. The package uses Google's deep learning library, TensorFlow, to construct a SAEHD (High Definition Styled AutoEncoder) model (trained over 65,000 iterations). The resulting model is essentially a moldable mask that was superimposed on the actor in the "destination" video (i.e., the video where our hired actor talks about essential oils in medicine). Only the video component was deepfaked—not the audio. ${ }^{5}$ The full technical details of the deepfake model are found in the Supplementary Materials (Section 1). This process emulates a malicious agent using a look-alike actor as a training set for the deepfake. The real video on essential oils and the deepfake thereof were used as treatments in Study 1.

Study 1 began with a series of screener and pre-treatment measures. Individuals were only included in the study if they provided consent, were over 18 years of age, were citizens of the United States, and were eligible to vote. Additionally, participants had to pass two attention checks and an audiovisual check to ensure that they were attentive and could view a video with sound. We then asked a series of questions on policy and political ideology that were used as pre-treatment covariates to improve the statistical precision of our treatment effect estimates.

\footnotetext{
${ }^{2}$ Lucid is an increasingly popular alternative to Amazon Mechanical Turk for social science survey research. Many wellknown findings have been replicated on Lucid, suggesting the platform is capable of providing high-quality data (Coppock \& McClellan, 2019, Peyton, Huber, \& Coppock, 2020). During the COVID-19 pandemic, Aronow et al. (2020) found that Lucid data can provide reliable data when researchers screen on attentiveness, which we do here. ${ }^{3}$ See https://osf.io/rqfz5/?view only=e2807b367a534262bb6c7aeb5727b999 for the pre-analysis plan.

4 The speech was composed using excerpts from Swaim \& Nussbaum (2016), a FiveThirtyEight article wherein Republican and Democratic speechwriters collaborated "to write a totally pandering bipartisan stump speech for an imaginary presidential candidate — one who espouses only positions that a majority of voters agree with" (p. 1). 5 This was a conscious choice since vocal impersonations are much easier and often more convincing than audio deepfakes. For example, one of the most widespread deepfakes in the wild (Jordan Peele as Obama) did not deepfake audio; Jordan Peele merely impersonated Barack Obama's voice.
} 
Participants were then shown a separate page with the following text: "On the next page, you will watch a brief political speech. You will then be asked about how you feel about the person making the speech and whether or not you would vote for them in the upcoming election." We randomized participants to receive either the real video or the deepfake version and either a warning or no warning in a 2x2 fully factorialized experiment. The warning read: "WARNING: Computer scientists are increasingly concerned about "DeepFake" videos. With DeepFakes, it's now possible to use a computer to convincingly manipulate videos of people to make them appear to say things they've never said.”'The four conditions are summarized in Table 1.

\section{Table 1: Description of Study 1 treatment arms}

\begin{tabular}{|c|c|c|}
\hline & $\begin{array}{c}\text { No Warning } \\
(\mathrm{N}=696)\end{array}$ & $\begin{array}{l}\text { Warning } \\
(\mathrm{N}=700)\end{array}$ \\
\hline $\begin{array}{c}\text { Real Video } \\
(\mathrm{N}=696)\end{array}$ & $\begin{array}{l}\text { (1) } \\
\text { Participants were shown a real } \\
\text { video of the actor stating that, if } \\
\text { elected, he would mandate doctors } \\
\text { to try using essential oils to treat } \\
\text { cancer before they are allowed to } \\
\text { use conventional medicine. } \\
(N=347)\end{array}$ & $\begin{array}{l}\text { (2) } \\
\text { Participants were shown the same video as } \\
\text { (1) but with a warning above the clip that } \\
\text { read "WARNING: Computer scientists are } \\
\text { increasingly concerned about "DeepFake" } \\
\text { videos. With DeepFakes, it's now possible } \\
\text { to use a computer to convincingly } \\
\text { manipulate videos of people to make them } \\
\text { appear to say things they've never said." } \\
(N=349)\end{array}$ \\
\hline $\begin{array}{c}\text { Deepfake } \\
\text { Video } \\
(\mathrm{N}=700)\end{array}$ & $\begin{array}{l}\text { (3) } \\
\text { This treatment arm was identical to } \\
\text { (1) except participants were shown } \\
\text { the deepfake version of the video. } \\
\qquad(N=349)\end{array}$ & $\begin{array}{l}\text { (4) } \\
\text { This treatment arm was identical to }(2) \\
\text { except participants were shown the } \\
\text { deepfake version of the video. } \\
(N=351)\end{array}$ \\
\hline
\end{tabular}

After viewing the video, we measured participants' perceived favorability of and intention to vote for the politician, their view on essential oils in medicine, whether they believed the video was real, and their overall confidence in other video footage of politicians speaking. Due to the risk of priming latent beliefs by asking participants if the video they just saw was real, ${ }^{6}$ we attempted to measure this outcome unobtrusively by asking whether they were convinced that the politician "believes what is said." We only explicitly ask participants whether they believed the video was deepfaked at the very end of the survey.

In this study, we found evidence that survey attrition varied significantly across treatment conditions (Pearson chi2 $(3)=11.3, \mathrm{p}=0.01) .4 .9 \%$ participants in the No Warning + Real Video condition did not finish the survey compared to $1.8 \%$ in the aggregate sum of the three remaining conditions. Because our pre-analysis plan did not explicitly stipulate how we would address differential attrition,

${ }^{6}$ Research on textual fake news found that asking participants about the accuracy of a specific piece of misinformation affected how likely they were to share that misinformation online (Pennycook et al., 2019; Pennycook, McPhetres et al., 2020). If priming accuracy can affect behavior, it has the potential to affect latent beliefs. 
we used Manski-type worst-case bounds (Manski, 2003) that require only that the support of the outcome is bounded when constructing our confidence intervals. ${ }^{7}$ These bounds are considered the gold-standard approach when analyzing experiments with attrition (Gerber and Green, 2012). Additional details are provided in Section 2 of the Supplementary Materials.

\section{Study 2: Real Politician ${ }^{8}$}

One weakness of Study 1 is that the extreme policy stance in the video could plausibly be genuine. Participants were not familiar with the politician (as he is fictional) and may not have had any expectation that the video they were watching could be deepfaked. McDonald (2019) empirically illustrated how people make use of prior knowledge of real-world politicians in online survey experiments and how studies using solely hypothetical politicians can produce misleading estimates of real-world political behavior. We therefore replicated the impact of warnings using a real politician in Study 2.

Figure 1: Screencaps of videos used in Study 1 (left) and Study 2 (right)

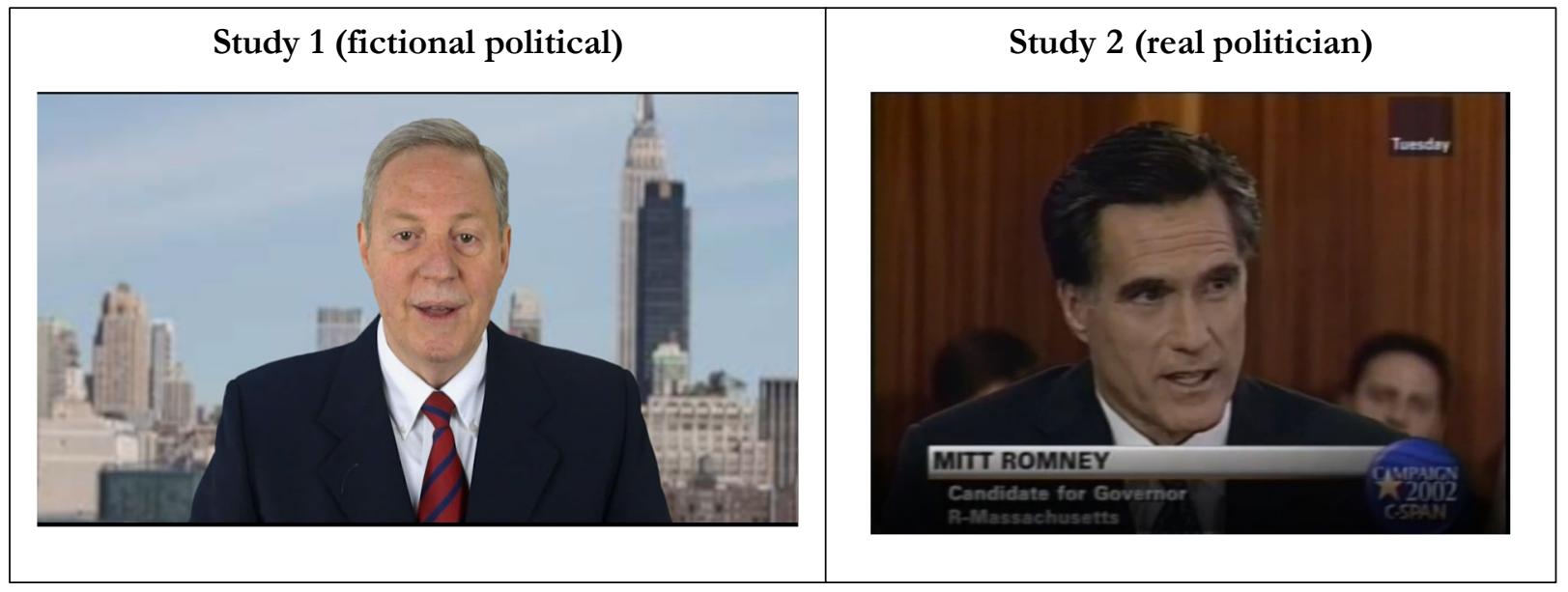

We identified a well-known politician who, at one point in his political career, had expressed a policy stance that was atypical given his party affiliation. Specifically, we found 2002 video footage of Republican Mitt Romney asserting, in a Massachusetts gubernatorial debate, that he would protect a woman's right to choose - an unusual stance for a Republican. ${ }^{9}$ For a still of this clip, see Figure 1, right. The design of this study was nearly identical to Study 1 with the following modifications.

First, Study 2 had only two conditions, Warning and No Warning (see Table 2 for a description of the two conditions), as we did not create a deepfake of Mitt Romney given ethical considerations. Second, our analysis was restricted to participants who knew Romney was a Republican pretreatment, as Romney's (formerly) pro-choice position would not appear surprising or controversial to those participants with no knowledge of Romney's party affiliation. Third, we collected additional pre-treatment covariates: Big Five personality traits, political knowledge, and political interest. We also included alternative measures of general trust in video footage of politicians speaking and

7 The "best" and "worst" cases used to estimate the CI are also covariate adjusted and use robust standard errors.

${ }^{8}$ See https://osf.io/rqfz5/?view only=e2807b367a534262bb6c7aeb5727b999 for the pre-analysis plan.

9 As of 2020, there are only 2 pro-choice Republicans in the Senate (Sussman, 2020). 
another measure of participants' belief in the content of the video. ${ }^{10}$ Study 2 was launched on November $1^{\text {st }}$ and again starting on November $8^{\text {th }}$ to measure differences in distrust immediately before and after the 2020 presidential election. In Study 2, we found no evidence of differential attrition: survey completion rates did not differ significantly across treatment arms.

Table 2: Description of Study 2 treatment arms

\begin{tabular}{|c|c|c|}
\hline & $\begin{array}{c}\text { No Warning } \\
(\mathrm{N}=966)\end{array}$ & $\begin{array}{l}\text { Warning } \\
(\mathrm{N}=959)\end{array}$ \\
\hline $\begin{array}{c}\text { Real Video } \\
(\mathrm{N}=1,925)\end{array}$ & $\begin{array}{l}\text { (1) } \\
\text { Participants watched a real video } \\
\text { of Mitt Romney saying “And I've } \\
\text { been very clear on that, I will } \\
\text { preserve and protect a woman's } \\
\text { right to choose and I'm devoted } \\
\text { and dedicated to honoring my } \\
\text { word..." }\end{array}$ & $\begin{array}{l}\text { (2) } \\
\text { Participants watched the same video as (1) } \\
\text { but with a warning above the clip that read } \\
\text { "WARNING: Computer scientists are } \\
\text { increasingly concerned about "DeepFake" } \\
\text { videos. With DeepFakes, it's now possible to } \\
\text { use a computer to convincingly manipulate } \\
\text { videos of people to make them appear to say } \\
\text { things thev've never said." }\end{array}$ \\
\hline
\end{tabular}

\section{Results and Discussion}

Discernment of Deepfakes

Our first question is whether subjects can discern between deepfakes and real videos. In Figure 2, we show that participants were unable to do so. The leftmost column in Figure 2 uses an outcome measure that asked participants how much they agreed with the statement "This video was doctored, manipulated and/or faked by a computer (i.e. it is a 'Deep Fake')" on a 7-point agree/disagree scale. Participants in the real video condition had a mean sentiment of -0.48 , which is nearly equidistant between "somewhat disagree" and "neither agree nor disagree." The mean sentiment of participants in the deepfake condition was not significantly different from that value (only 0.07 points higher). ${ }^{11}$ As shown in the remaining two columns, treatment effects were even smaller across alternative measures of disbelief. In other words, a well-made deepfake video is unlikely to be detected by the naked eye of a typical American voter. In fact, it appears that Americans are somewhat confident that a given political video is not manipulated by a computer-even when it is. ${ }^{12}$

10 To ensure we are not priming participants, we included a free response question asking participants to name three facts about Romney. But since free response questions are answered at very low rates (e.g. Luskin \& Bullock, 2011), we subsequently included a true/false question matrix with Romney facts, which included his stance on abortion.

11 We do not believe that this is a false negative resulting from the bounds. Without bounds, the p-value is 0.57.

12 The deepfake we tested may be of higher quality than what a malicious agent can currently create. In reality, the politician would not be the one delivering the deepfaked message as he did in our study; the message would be delivered by an impersonator with highly similar facial features. The deepfake software would then swap faces of the impersonator and the politician. We do not believe this is a major concern: considering that the quality of deepfake software continues to rapidly improve, it is unlikely that this level of deepfake quality will long remain unattainable for malicious agents. The first confirmed real-world political deepfake appeared to have a similar level of visual quality to our video in Study 1 ; the 
Figure 2: Voters were unable to discriminate between a real video and a deepfake (Study 1)

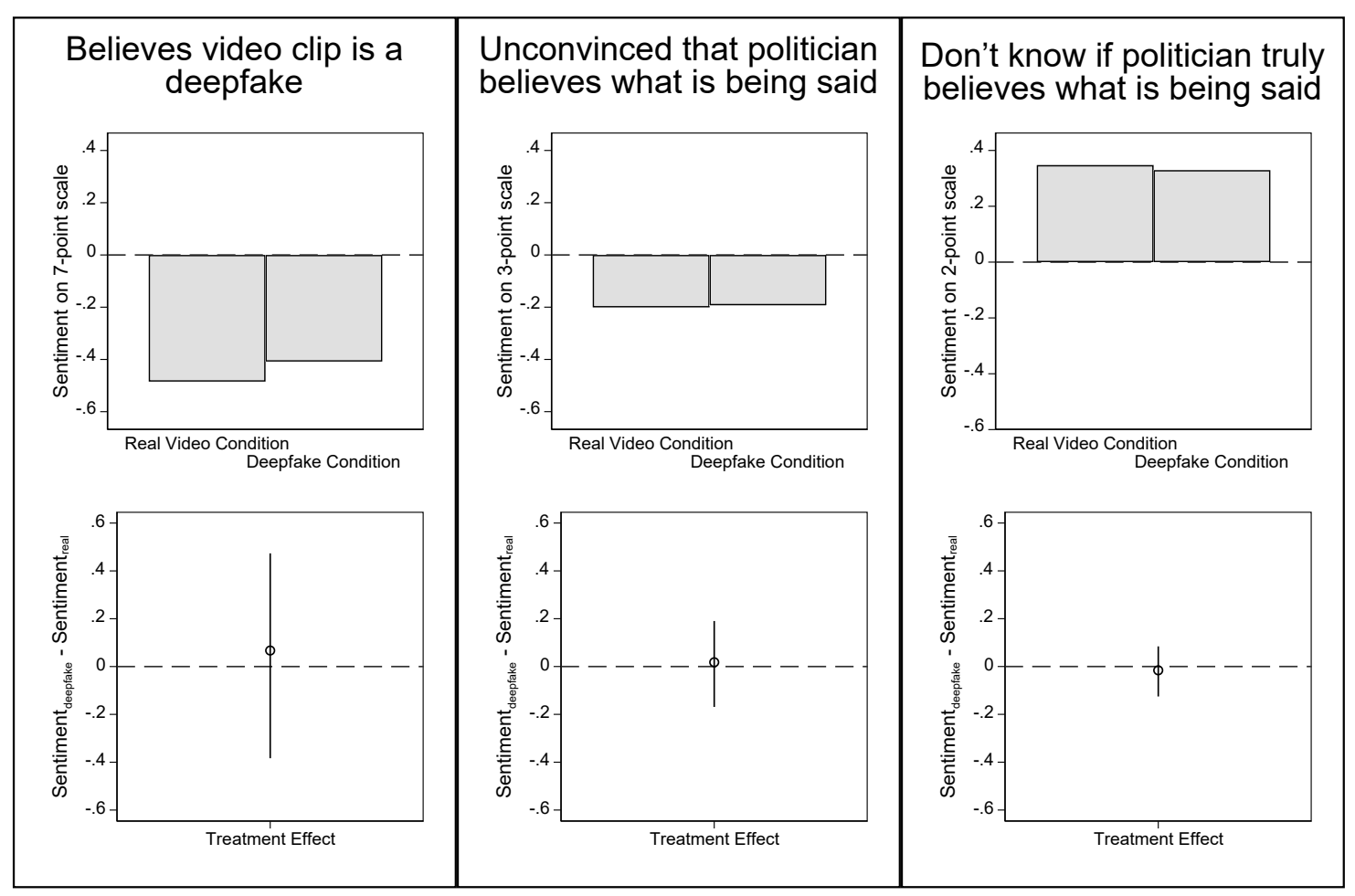

This figure includes only those participants who did not receive a deepfake warning. The top row illustrates covariate unadjusted mean sentiment in each treatment arm for each outcome. The bottom row shows covariate-adjusted treatment effects with Manski bounds used to calculate 95\% confidence intervals. The three columns represent three outcomes (from left to right): 1) do you believe the video clip is a deepfake? [7-point scale from -3= "strongly disagree" to 3= "strongly agree"]; 2) how convinced are you that the politician believes what is being said? [3-point scale from 1="not at all convinced" to -1="very convinced"]; and 3) I don't know if the politician truly believes what is said [binary variable].

\section{Impact of Deepfake Warnings}

We now turn to our central question: how do deepfake warnings affect evaluations of real and fake political videos? In the leftmost column of Figure 3, we see that when the warning was randomly added to a deepfake video, participants were 0.5 points more likely to believe that the video they were watching was fabricated $(\mathrm{p}<0.001) .{ }^{13}$ It is worth noting that this treatment effect was not driven by people affirmatively identifying the deepfake. When participants watched a deepfaked video without a warning, they were fairly confident that what they were watching was not a deepfake (-0.4 points on our 7-point scale); when the warning was added, they became more uncertain about whether the video was a deepfake (0.1 points). ${ }^{14}$

Bharatiya Janata Party (BJP) in India disseminated a video deepfaked to make it appear that the BJP President was delivering a speech in a different language (Christopher, 2020).

${ }^{13}$ For brevity, we use only one outcome variable (do you believe the video clip is a deepfake?) in this analysis. We see similar but smaller treatment effects using the two less-granular outcome variables (Figures OA1a and OA1b).

14 This is verified by comparing the distributions of responses in the two warning conditions; see Figure OA2. 
Figure 3: Warnings induced disbelief in accompanying video regardless of whether the video is real or fake

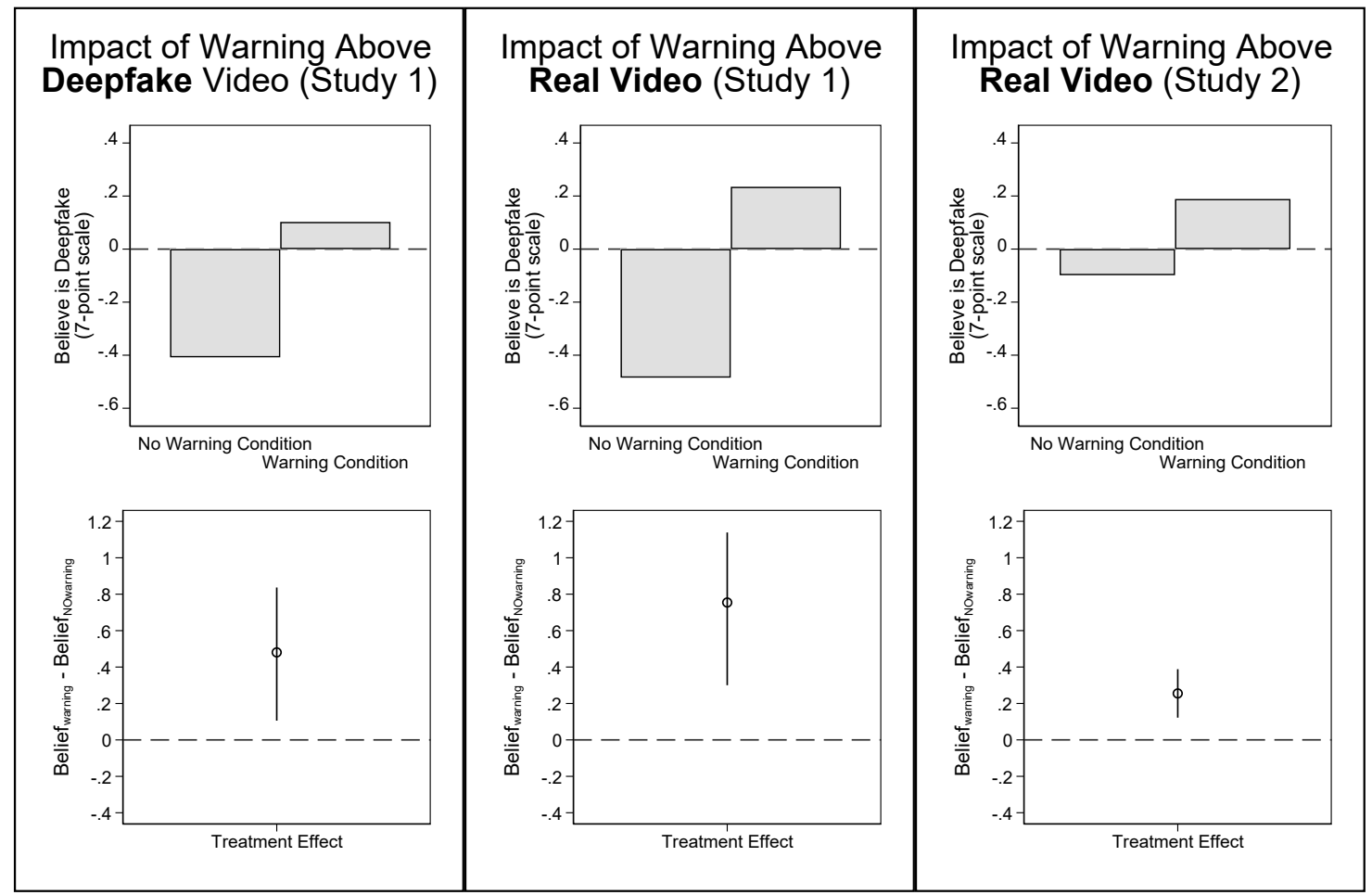

The top row illustrates covariate unadjusted mean sentiment in each treatment arm, while the bottom row shows the resulting treatment effects with 95\% confidence intervals. The leftmost column illustrates the impact of a warning added above a deepfake video; the remaining columns illustrate the impact of a warning added above a real video. All three columns measure impact in terms of the same outcome: do you believe the video clip is a deepfake? The two leftmost treatment effect graphs use Manski bounds to calculate $95 \%$ confidence intervals. The rightmost treatment effect graph uses covariate-adjusted standard errors from an OLS regression, as there was no differential attrition.

We see a comparable treatment effect $(.75$ points $[\mathrm{p}<0.001])$ when the warning was randomly added to a real video of the same content (see the middle column in Figure 3). The interaction of the deepfake treatment and the warning treatment is not statistically significant. ${ }^{15}$ Rather than helping participants detect deepfake videos, the warning instead caused participants to disbelieve whatever video they were watching - real or fake. This interaction effect is directionally opposite from the normative ideal: adding warnings to videos made participants more likely to disbelieve the real video rather than successfully identify the deepfake.

We find similar effects with the real video of Romney in Study 2. The results are summarized in the rightmost column of Figure 3. As before, we see that a generic warning about deepfakes induced participants to disbelieve real videos_an effect of 0.26 points on a 7 -point scale $(\mathrm{p}<0.001)$. One notable difference between the results of Study 1 and Study 2 is the baseline levels of disbelief.

${ }^{15}$ Even without bounds, the p-value is 0.13 and the effect is in the opposite direction from what would be desired. 
Whereas Study 1 participants were relatively confident that the warning-free video of the fictional politician was not a deepfake (-0.4 points on our 7-point scale), participants in the same condition in Study 2 were more uncertain (- 0.1 points). This may be because participants in Study 2 were familiar with Mitt Romney and thus held some prior belief as to what Romney could plausibly say. ${ }^{16}$ In Study 1, participants had no prior beliefs about the fictional politician, so they likely had no reason to suspect that the video they were watching could be manipulated.

Pooling the data from the two studies and including a study fixed effect, we find that warnings increased participants' belief that the video was fabricated by 0.40 points on a 7 -point scale $[\mathrm{p}<0.001] .{ }^{17}$ Warnings also increased how unconvinced participants were that the politician actually believed what he was saying by 0.13 points (on a 3-point scale) $[\mathrm{p}<0.001]$. Finally, warnings also increased the rate at which participants said that they "didn't know whether the politician believed what was said in the video" (a binary variable) by 6.3 percentage points $(\mathrm{p}=0.001) .{ }^{18}$

We also evaluated whether warnings can affect how participants internalize the content of the video. Namely, do generic warnings change what information participants associate with the politician in the video ${ }^{19}$ Towards the end of Study 2, we asked participants to name three facts about Romney in an open-ended question. As is typical of open-ended political knowledge questions (e.g., Luskin \& Bullock, 2011), we found a very low base rate of participants naming policy stances. Most of the responses were personally descriptive facts (e.g., "He is Republican", "He ran for president"), followed by subjective assessments ("honest", "traitor"), with only $2 \%$ of responses explicitly referencing Romney's stance on abortion. ${ }^{20}$ We found that warnings did not significantly change how likely participants were to mention abortion $(\mathrm{p}=0.68)$, but there was a major shift in what participants perceived Romney's position on abortion to be. The warning caused a 3.0 percentage point drop in the percentage of participants who associated Romney with a pro-choice position $(\mathrm{p}=0.001) .{ }^{21}$ An alternative measure of this outcome asked participants in a close-ended question if Romney had ever "supported women's access to abortion"; we found that adding a warning decreased the likelihood that participants marked "true" by 14.2 percentage points $(\mathrm{p}<0.001)$.

Finally, we assessed whether warnings have broader effects beyond the specific videos included in the studies: do generic warnings cause Americans to doubt political footage that they may view in

$16 \mathrm{We}$ also find suggestive evidence in favor of this hypothesis in Study 2. Before participants watched the treatment video, participants rated how familiar they were with Romney. In the No Warning condition only, participants who were less familiar with Romney ("somewhat familiar" or "never heard of") were 0.2 points less likely (on our 7-point scale) to believe that the video was a deepfake as compared to participants who were "very familiar" with Romney $(p<0.05)$.

17 We exclude Study 1 participants randomized to the deepfake condition in the reported specification, but the results do not change meaningfully when we include the deepfake condition.

18 Warnings also appear to have some impact on favorability and intention to vote for the politician featured in the video clip. Warnings decrease intention to vote for the politician by 4.2 percentage points $(\mathrm{p}<0.05)$, with little difference in effect sizes across studies. For more details, please see Section 4 of the Supplementary Materials.

${ }_{19}$ A treatment effect on belief in the veracity of a particular video does not necessarily imply that the video affects latent beliefs about the politician. Namely, an apathetic voter may view the video of Romney saying he is pro-choice and believe the video is real, but never associate Romney with a pro-choice position-not because they are skeptical of the source of the information, but because the information itself is uninteresting or unimportant to the participant. ${ }^{20}$ We coded statements that were likely about abortion policy but were nevertheless too vague (e.g., "He supports women", "Choose", "For Women's Rights") as "other." Additional details are provided in Table OA2.

21 The estimand here is rather complex in that the correct answer is that Romney supported abortion rights in the past but does not currently. Most responses were written in present tense, but it is not clear as to whether a "he is prochoice" answer is an artifact of participant succinctness or if it accurately reflects their belief about Romney. 
the future? We asked participants how much confidence they had in footage of political speeches found online and on broadcast television. As seen in Figure 4, warnings did not impact trust in footage found on TV or online with precisely estimated null effects. ${ }^{22}$ Pooling the studies, we found a difference of $-0.03(p=0.35)$ on trust in footage online and $0.03(p=0.46)$ on trust in footage on TV. It is worth emphasizing that the impacts of warnings on trust online are consistently negative, though small, but the treatment was just a single warning. It is possible that higher dosages of rhetoric about deepfakes may erode trust in direct video footage of politicians speaking.

\section{Figure 4: Warnings had no impact on trust in future videos found on TV and online}
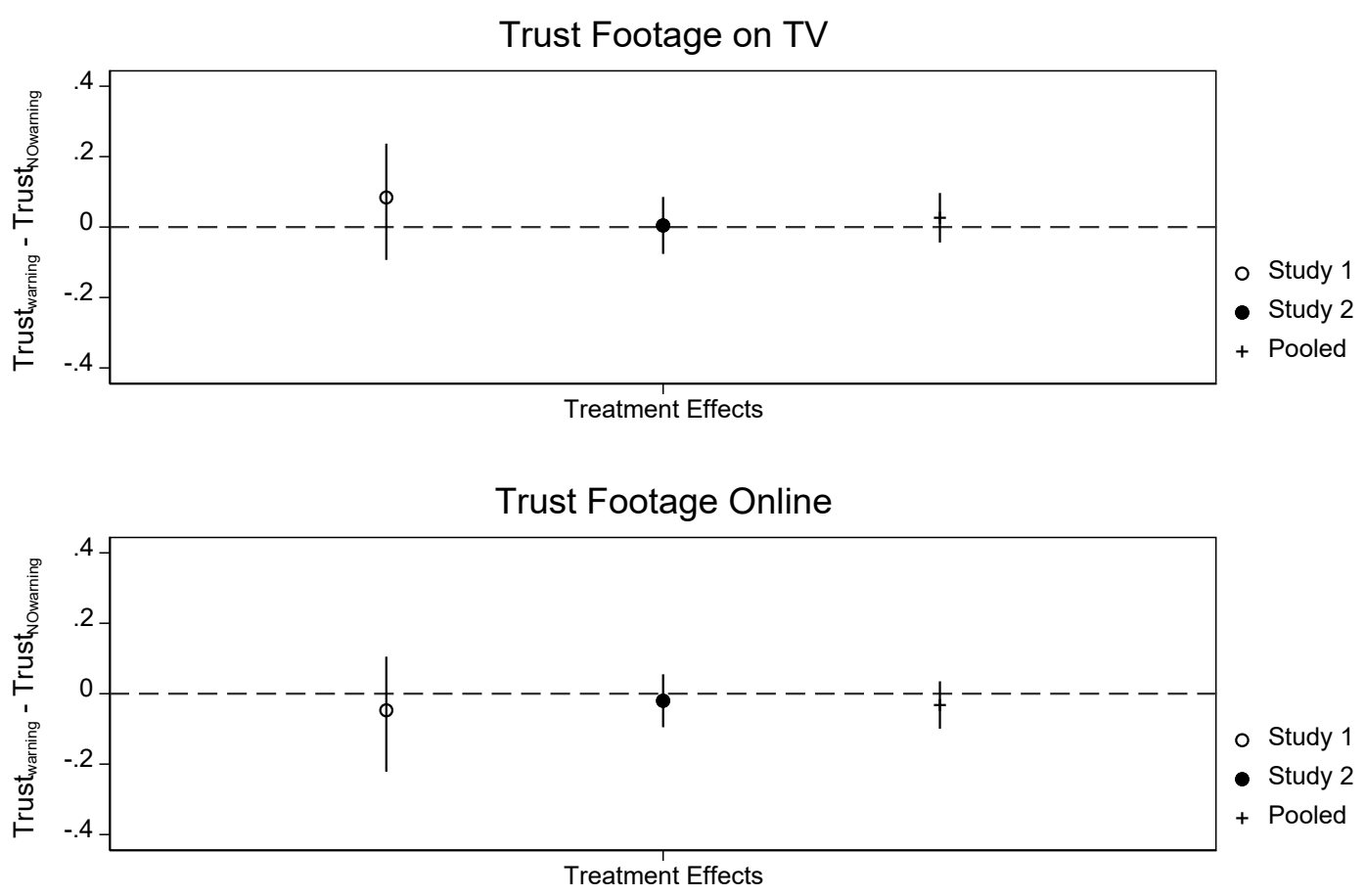

The outcome measure has the same wording across both studies: "When you see videos of politicians making controversial statements [on television news/online], how confident are you that they haven't been faked, altered, or manipulated?" Responses range from $0=$ "not at all confident" to $4=$ "completely confident." The two panels illustrate covariate-adjusted treatment effects. Study 1 treatment effects use Manski bounds to calculate 95\% confidence intervals. The confidence intervals in Study 2 are estimated using covariate-adjusted standard errors from an OLS regression, as there was no differential attrition. The pooled estimate is estimated using a regression with shared covariates; it excludes all participants in the deepfake condition in Study 2.

Our findings add to the growing body of literature on textual misinformation, which has consistently found that warnings may help readers reject misinformation but may have the undesirable backlash of increasing Americans' disbelief in true news stories (e.g., Pennycook, Bear et al., 2020; Clayton et al., 2020). We find strong evidence that warnings increase disbelief in accompanying video clipsregardless of whether the video is fake or real. This is particularly problematic as the warnings driving this effect are vague. The warnings did not say that the accompanying video was fake; they

22 An alternative wording of the same outcome ("Imagine you saw a video of a politician saying something controversial on [television news/Facebook or Twitter]. How likely are you to believe that the politician actually said what you see in the video?’) was used in Study 2 and produced very similar treatment effects. See Table OA5. 
simply stated that deepfakes exist and are challenging to spot, which is something that an American might hear on a news program. Such a light nudge nevertheless induced people to disbelieve a video that revealed a little-known but real policy stance of a real-world politician.

\section{Heterogenous Treatment Effects}

We also examined heterogeneous treatment effects, but as we noted in both pre-analysis plans, we were underpowered for most of these analyses. We give a brief overview of the most noteworthy results here (see Sections 3-4 of the Supplementary Materials for full results). First, we found some evidence that participants surveyed before the election have higher levels of distrust in political videos than participants surveyed after the election $(\mathrm{p}<.05)$. Second, we found no evidence in Study 2 in support of motivated reasoning; Republicans, when treated with a warning, were not more likely than Democrats to reject the idea that Romney was ever pro-choice (interaction coefficient $\mathrm{p}$ values $>0.43$ ). Instead, our results are consistent with the "lazy not biased" model of political (mis)information consumption (Pennycook \& Rand, 2019).

\section{Conclusions}

Our results illustrate that deepfakes pose a significant problem for democracies not simply through the spread of misinformation but rather through the delegitimization of true information. Attempts to warn the public about deepfakes may in fact make matters worse. The most important implication of our findings is that elites and social media platforms should take great care in their attempts to educate the public. We show that indiscriminate warnings erode trust, and it is thus imperative that other approaches be the subject of future research.

\section{References}

Aronow, P. M., Kalla, J., Orr, L., \& Ternovski, J. (2020). Evidence of Rising Rates of Inattentiveness on Lucid in 2020. Working Paper.

Barabas, J., Jerit, J., Pollock, W., \& Rainey, C. (2014). The question (s) of political knowledge. American Political Science Review, 840-855.

Barari, S., Lucas, C., \& Munger, K. (2021). Political Deepfake Videos Misinform the Public, But No More than Other Fake Media. Working Paper.

Clayton, K., Blair, S., Busam, J.A., Forstner, S., Glance, J., Green, G., Kawata, A., Kovvuri, A., Martin, J., Morgan, E et al. (2020). Real solutions for fake news? Measuring the effectiveness of general warnings and fact-check tags in reducing belief in false stories on social media. Political Behavior, 42, 1073-1095.

Coppock, A., \& McClellan, O. A. (2019). Validating the demographic, political, psychological, and experimental results obtained from a new source of online survey respondents. Research \& Politics, 6(1), 2053168018822174.

Christopher, N. (2020). We've Just Seen the First Use of Deepfakes in an Indian Election Campaign. Vice News. February. Retrieved from https://www.vice.com/en/article/igedjb/the-first-useof-deepfakes-in-indian-election-by-bjp

Dack, S. (2019). Deep Fakes, Fake News, and What Comes Next. The Henry M. Jackson School of International Studies News. University of Washington. March 20. Retrieved from https://jsis.washington.edu/news/deep-fakes-fake-news-and-what-comes-next/

Dobber, T., Metoui, N., Trilling, D., Helberger, N., \& de Vreese, C. (2020). Do (microtargeted) deepfakes have real effects on political attitudes?. The International Journal of Press/Politics, 1940161220944364. 
Gerber, A. S., \& Green, D. P. (2012). Field Experiments: Design, Analysis, and Interpretation. New York: W.W. Norton.

Lazer, D. M., Baum, M. A., Benkler, Y., Berinsky, A. J., Greenhill, K. M., Menczer, F., Metzger, M., Nyhan, B., Pennycook, G., Rotschild, D. et al. (2018). The science of fake news. Science, 359(6380), 1094-1096.

Lenz, G. S. (2013). Follow the leader?: how voters respond to politicians' policies and performance. University of Chicago Press.

Luskin, R. C., \& Bullock, J. G. (2011). “Don't know” means “don't know”: DK responses and the public's level of political knowledge. The Journal of Politics, 73(2), 547-557.

Manski, C. F. (2003). Partial identification of probability distributions. Springer Science \& Business Media.

McDonald, J. (2019). Avoiding the Hypothetical: Why "Mirror Experiments" are an Essential Part of Survey Research. International Journal of Public Opinion Research, 32(2), 266-283.

O'Sullivan (2019). House Intel chair sounds alarm in Congress' first hearing on deepfake videos, CNN Business. June 13. Retrieved from https://www.cnn.com/2019/06/13/tech/deepfakecongress-hearing/index.html

Pennycook, G., Bear, A., Collins, E. T., \& Rand, D. G. (2020). The implied truth effect: Attaching warnings to a subset of fake news headlines increases perceived accuracy of headlines without warnings. Management Science.

Pennycook, G., Epstein, Z., Mosleh, M., Arechar, A. A., Eckles, D., \& Rand, D. G. (2019). Understanding and reducing the spread of misinformation online. Working Paper.

Pennycook, G., McPhetres, J., Zhang, Y., Lu, J. G., \& Rand, D. G. (2020). Fighting COVID-19 misinformation on social media: Experimental evidence for a scalable accuracy-nudge intervention. Psychological science, 31(7), 770-780.

Pennycook, G., \& Rand, D. G. (2019). Lazy, not biased: Susceptibility to partisan fake news is better explained by lack of reasoning than by motivated reasoning. Cognition, 188, 39-50.

Peyton, K., Huber, G. A., \& Coppock, A. (2020). The Generalizability of Online Experiments Conducted During the COVID-19 Pandemic. Working Paper.

Sussman, A. L. (2020). The Loneliness of the Pro-Choice Republican Woman. The New Yorker. October. Retrieved from https://www.newyorker.com/news/us-journal/the-loneliness-ofthe-pro-choice-republican-woman

Swaim, B. \& Nussbaum, J. (2016). The Perfect Presidential Stump Speech. FiveThirtyEight. November. Retrieved from https://projects.fivethirtyeight.com/perfect-stump-speech/

Vaccari, C., \& Chadwick, A. (2020). Deepfakes and disinformation: Exploring the impact of synthetic political video on deception, uncertainty, and trust in news. Social Media and Society, 6(1), 2056305120903408.

Van Duyn, E., \& Collier, J. (2019). Priming and fake news: The effects of elite discourse on evaluations of news media. Mass Communication and Society, 22(1), 29-48.

Villarreal, D. (2021). Parler Users Call Trump's Concession Video 'Deep Fake,' and Worry He'll 'Sell Us Out' Newsweek, January. Retrieved from https://www.newsweek.com/parler-users-calltrumps-concession-video-deep-fake-worry-hell-sell-us-out-1559895

Vosoughi, S., Roy, D., \& Aral, S. (2018). The spread of true and false news online. Science, 359(6380), 1146-1151.

Wittenberg, C., Zong, J., \& Rand, D. (2020). The (Minimal) Persuasive Advantage of Political Video over Text. Working Paper. 